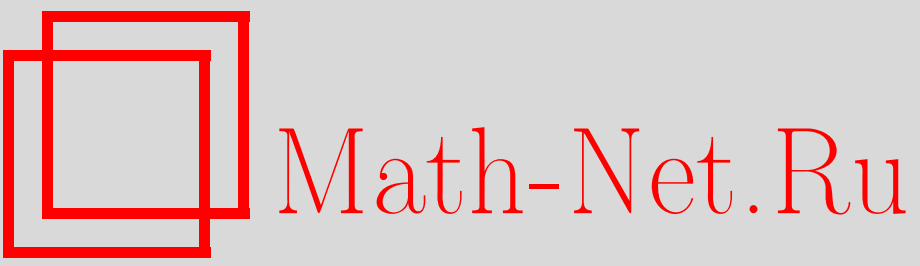

С. А. Пастон, Е. В. Прохватилов, В. А. Франке, Калибровочно-инвариантная регуляризация квантовой теории поля на световом фронте, ТМФ, 2004, том 139, номер 3, 429-448

DOI: https://doi.org/10.4213/tmf70

Использование Общероссийского математического портала Math-Net.Ru подразумевает, что вы прочитали и согласны с пользовательским соглашением

http://www.mathnet.ru/rus/agreement

Параметры загрузки:

IP: 3.91 .87 .62

26 апреля 2023 г., 13:55:05 
ТЕОРЕТИЧЕСКАЯ

И МАТЕМАТИЧЕСКАЯ

ФИЗИКА

Том 139, № 3

июнь, 2004

(C) 2004 г. С. А. Пастон ${ }^{*}$, Е. В. Прохватилов ${ }^{\dagger}$, В. А. Франке

\section{КАЛИБРОВОЧНО-ИНВАРИАНТНАЯ РЕГУЛЯРИЗАЦИЯ КВАНТОВОЙ ТЕОРИИ ПОЛЯ НА СВЕТОВОМ ФРОНТЕ}

Кратко описаны проблемы, присущие гамильтонову подходу к квантованию калибровочных полей на световом фронте при ограничении пространства-времени неравенством $\left|x^{-}\right| \leqslant L$ и периодических граничных условиях для полей по координате $x^{-}$(метод DLCQ). В этих рамках рассмотрена калибровочно-инвариантная ультрафиолетовая регуляризация с помощью перехода к решетке по поперечным координатам. Остающиеся ультрафиолетовые расходимости по продольному импульсу $p_{-}$устраняются калибровочно-инвариантной конечно-модовой регуляризацией. Оказывается, что при введенной регуляризации канонический формализм на световом фронте не содержит обычных наиболее сложных связей второго рода между нулевыми и ненулевыми модами полей. Описанная схема может использоваться как для регуляризации обычной калибровочной теории, так и для калибровочно-инвариантной формулировки эффективных низкоэнергетических моделей на световом фронте. Из-за нарушения явной лоренц-инвариантности в рассматриваемом формализме возникают трудности, связанные с определением вакуумного состояния. Эти трудности обсуждаются, в частности, в связи с проблемой перехода к пределу непрерывного пространства.

Ключевые слова: калибровочные поля, световой фронт, гамильтонов подход, поперечная решетка.

\section{1. ВВЕДЕНИЕ}

Каноническая формулировка теории поля в координатах светового фронта $(\mathrm{C} \Phi) x^{ \pm}=$ $\left(x^{0} \pm x^{3}\right) / \sqrt{2}, x^{1}, x^{2}$, где $x^{0}, x^{1}, x^{2}, x^{3}$ - лоренцевы координаты, была предложена Дираком [1]. В этом подходе координата $x^{+}$играет роль времени, так что канонический формализм строится на гиперплоскости $x^{+}=$const. Преимущества этой формулировки связаны с упрошением описания физического вакуума благодаря неотрицательности импульса $P_{-}$(генератора сдвига вдоль оси $x^{-}$), который на СФ имеет простое, квадратичное по полям, выражение. Как следствие низшее собственное состояние оператора

\footnotetext{
* Санкт-Петербургский государственный университет, Санкт-Петербург, Россия. E-mail: paston@pobox.spbu.ru; franke@pobox.spbu.ru

${ }^{\dagger}$ НИИ физики, Санкт-Петербургский государственный университет, Санкт-Петербург, Россия. E-mail: Evgeni.Prokhvat@pobox.spbu.ru
} 
$P_{-}$описывает как физический вакуум, так и "математический" вакуум теории возмущений. Используя фоковское пространство над этим вакуумом, можно решать стационарное уравнение Шредингера с гамильтонианом $P_{+}$(оператором эволюции по $x^{+}$) с целью непертурбативного расчета спектра связанных состояний. Это так называемый гамильтонов подход на СФ.

С другой стороны, плоскость $x^{+}=$const - характеристическая поверхность дифференциальных уравнений поля. Поэтому не очевидно, что квантование на этой поверхности эквивалентно квантованию в обычных лоренцевых координатах [2]-[4]. В частности, в каноническом формализме на СФ присутствуют сингулярности при $p_{-}=0$. Это требует введения, кроме обычной ультраффиолетовой регуляризации, еще и регуляризации особенностей при $p_{-} \rightarrow 0$, что может нарушить лоренцеву или калибровочную симметрии даже в пределе снятия регуляризации [3].

Обычно эту регуляризашию вводят одним из следуюших простейших способов:

а) путем обрезания импульса

$$
\left|p_{-}\right| \geqslant \varepsilon, \quad \varepsilon>0 ;
$$

б) путем ограничения координаты

$$
-L \leqslant x^{-} \leqslant L
$$

и выбора периодических граничных условий по $x^{-}$для всех полей.

Эти способы удобны для гамильтонова подхода на СФ, однако они оба не инвариантны относительно лоренцевых преобразований. В случае калибровочных теорий калибровочно-инвариантна только регуляризация "б".

Проблема построения гамильтониана на С $\Phi$, ведушего к результатам, которые эквивалентны возникающим в обычной лоренц- и калибровочно-инвариантной формулировке теории, оказывается достаточно сложной. Тем не менее в рамках теории возмушений во всех порядках эту проблему можно решить как для некалибровочных [5], так и для калибровочных [6] теорий, используя регуляризацию "а" и специальные методы, позволяюшие восстановить нарушенные симметрии в пределе снятия регуляризации. Но возникаюший гамильтониан оказывается весьма сложным, особенно в случае калибровочных теорий, причем он содержит много неизвестных параметров.

Способ "б" ведет к дискретизации спектра оператора $P_{-}\left(p_{-}=\pi n / L, n \in \mathbb{Z}\right)$ и в литературе носит название дискретизованное квантование на СФ (DLCQ) [7]. Фурье-компоненты полей по $x^{-}$, отвечающие $p_{-}=0$ (и именуемые обычно нулевыми мо$\partial а м u)$, оказываются зависимыми переменными и должны быть выражены через ненулевые моды посредством решения канонических связей второго рода [8]-[10]. Такие связи обычно нелинейны по полям, и их решение представляет трудности, не говоря уже о проблеме расстановки операторов в соответствуюших этим связям квантовых выражениях. Обычно данное построение проводится в непрерывном пространстве-времени. При этом ввести калибровочно-инвариантную ультрафиолетовую регуляризацию достаточно трудно. Поэтому чаше всего ограничиваются простейшей калибровочно-неинвариантной ультрафиолетовой регуляризацией. Такой подход кратко излагается в разделах 2,3 данной работы. 
С другой стороны, в качестве калибровочно-инвариантной ультрафиолетовой регуляризации неабелевых калибровочных теорий хорошо известно введение пространственно-временной решетки [11]. (В непрерьвном пространстве можно использовать также калибровочно-инвариантную регуляризацию, предложенную в работе [12], но не ясно, каким образом в ее рамках развивать канонический формализм на СФ.) Для формулировки теории на СФ применяется решетка только по поперечным координатам $x^{1}, x^{2}$. При этом удобно использовать вариант теории, в котором действие полиномиально по полям [13]. Такая регуляризация, разумеется, нарушает лоренц-инвариантность, и можно лишь надеяться, что последняя восстанавливается при предельном переходе к непрерывному пространству.

В разделах 4,5 настояшей работы рассматривается каноническая формулировка на СФ калибровочных теорий, регуляризованных калибровочно-инвариантным образом путем дискретизации "б" импульса $p_{-}$, введения решетки по поперечным координатам и использования так назьваемой конечно-модовой регуляризации по $p_{-}$. Последняя основана на обрезании мод оператора ковариантной производной $D_{-}$, фигурируюших в разложении переменных поля на решетке по собственным функциям этого оператора. Указанные переменные поля суть решеточный аналог поперечных компонент обычного калибровочного поля. Они описываются комплексными матрицами, отнесенными к ребрам решетки, и допускают вышеупомянутую конечно-модовую регуляризацию (для фермионных полей аналогичная регуляризация применялась в ряде работ; см., например, [14]).

Интересно, что в рамках такой формулировки удается избежать сложных канонических связей второго рода, обычно возникаюших в теории на СФ в непрерывном пространстве. Это значительно упрошает каноническую процедуру квантования. Однако отсутствие явной лоренц-инвариантности в рассматриваемой схеме затрудняет исследование связи полученной квантовой теории с обычной лоренц-ковариантной формулировкой. В частности, существует проблема описания квантового вакуума, поскольку выясняется, что до снятия регуляризации низшее состояние оператора $P_{-}$может не совпадать с низшим состоянием гамильтониана $P_{+}$. Этот вопрос обсуждается в конце раздела 5 .

С другой стороны, если начинать с полуфеноменологического (эффективного) гамильтонова подхода на С $\Phi$, также используюшего решетку по поперечным координатам и те же матричные переменные на ребрах решетки [15], то данньй формализм дает полностью калибровочно-инвариантную формулировку такого подхода. В этом случае проблему вакуума можно решить, модифицируя соответствуюшим образом гамильтониан $P_{+}$.

\section{2. КЛАССИЧЕСКАЯ ТЕОРИЯ КАЛИБРОВОЧНЫХ ПОЛЕЙ В КООР ДИНАТАХ СВЕТОВОГО ФРОНТА}

Рассмотрим сначала, как можно сформулировать калибровочную теорию поля на $\mathrm{C} \Phi$, используя непрерывное пространство-время при введении периодических граничных условий по координате $x^{-}$. При этом пока не будем фиксировать способ ультрафиолетовой регуляризации и ограничимся простейшей неабелевой калибровочной группой 
$S U(2)$.

Введем основные обозначения. Координаты С $\Phi x^{\mu}=\left(x^{+}, x^{-}, x^{\perp}\right), x^{\perp} \equiv x^{k}, k=$ 1,2 , соответствуют метрическому тензору $g_{\mu \nu}$ с ненулевыми компонентами $g_{+-}=$ $g_{-+}=-g_{11}=-g_{22}=1 ; A_{\mu}^{a}(x)$ - вектор-потенциал калибровочного поля, $a=1,2,3-$ индекс присоединенного представления калибровочной группы; $F_{\mu \nu}^{a} \equiv \partial_{\mu} A_{\nu}^{a}-\partial_{\nu} A_{\mu}^{a}+$ $g \varepsilon^{a b c} A_{\mu}^{b} A_{\nu}^{c}, \varepsilon^{a b c}$ - полностью антисимметричный тензор $\left(\varepsilon^{123}=1\right) ; g$ - константа взаимодействия; для любого изовектора $\widehat{O} \equiv O^{a} \tau^{a} / 2 ; \tau^{a}$ - матрицы Паули. Дираковские биспиноры

$$
\Psi^{i}(x) \equiv\left(\begin{array}{c}
\chi^{i}(x) \\
\xi^{i}(x)
\end{array}\right)
$$

где $i$ - индекс фундаментального представления калибровочной группы, являются грассмановыми (антикоммутируюшими) переменными и соответствуют следуюшему представлению $\gamma^{\mu}$-матриц Дирака:

$$
\begin{aligned}
\gamma^{+}=i \sqrt{2}\left(\begin{array}{cc}
0 & 0 \\
1 & 0
\end{array}\right), & \gamma^{-}=i \sqrt{2}\left(\begin{array}{cc}
0 & -1 \\
0 & 0
\end{array}\right), \\
\gamma^{k}=i\left(\begin{array}{cc}
-\sigma_{k} & 0 \\
0 & \sigma_{k}
\end{array}\right), & \gamma^{0}=i\left(\begin{array}{cc}
0 & -1 \\
1 & 0
\end{array}\right),
\end{aligned}
$$

где $\sigma_{k}-$ спиновые матришы Паули. Введем также обозначения: $\Psi^{+}-$эрмитово сопряженньй (э.с.) биспинор,

$$
\widehat{D}_{\mu} \equiv \partial_{\mu}-i g \hat{A}_{\mu}, \quad \bar{D} \equiv \widehat{D}_{k} \sigma_{k}, \quad\left(D_{\mu}\right)^{a c} \equiv \delta^{a c} \partial_{\mu}+g \varepsilon^{a b c} A_{\mu}^{b}
$$

“Нулевая мода" $(f)_{0}$ функции $f\left(x^{-}\right)$есть

$$
(f)_{0} \equiv \frac{1}{\sqrt{2 L}} \int_{-L}^{L} d x^{-} f\left(x^{-}\right)
$$

Калибровочное преобразование действует следуюшим образом:

$$
\Psi \rightarrow \Omega \Psi, \quad \hat{A}_{\mu} \rightarrow \Omega \hat{A}_{\mu} \Omega^{-1}+\frac{i}{g} \Omega \partial_{\mu} \Omega^{-1}, \quad \Omega=e^{i \omega^{a} \tau^{a} / 2} .
$$

В этих обозначениях рассматриваемая теория определяется калибровочно-инвариантной плотностью лагранжиана:

$$
\mathcal{L}=-\frac{1}{4} F_{\mu \nu}^{a} F^{\mu \nu a}+\Psi^{+} \gamma^{0}\left(i \widehat{D}_{\nu} \gamma^{\nu}-M\right) \Psi
$$

где $M$ - массовый параметр фермионов. В координатах СФ получаем

$$
\begin{aligned}
\mathcal{L}= & \frac{1}{2} F_{+-}^{a} F_{+-}^{a}+F_{+k}^{a} F_{-k}^{a}-\frac{1}{2} F_{12}^{a} F_{12}^{a}+ \\
& +i \sqrt{2} \chi^{+} \widehat{D}_{+} \chi+i \sqrt{2} \xi^{+} \widehat{D}_{-} \xi+\left\{i \xi^{+}(\bar{D}-M) \chi+\text { э.c. }\right\}
\end{aligned}
$$


Калибровочно-инвариантные периодические граничные условия на поля соответствуют их периодичности с точностью до калибровочного преобразования. Всегда можно перейти к такой калибровке [8], в которой потенциалы $A_{\mu}^{a}$ точно периодичны по $x^{-}$, а фермионные поля $\Psi^{i}$ либо периодичны, либо антипериодичны. В этой калибровке остаточная калибровочная группа симметрии отвечает периодическим либо антипериодическим матрицам $\Omega(x)$. Такой калибровочной свободы достаточно [8], чтобы удовлетворить дополнительному условию

$$
\partial_{-} A_{-}^{a}=0, \quad A_{-}^{a} \equiv v^{a}\left(x^{\perp}, x^{+}\right) .
$$

Оно отличается от обычной “светоподобной” калибровки $A_{-}^{a}=0$ наличием нулевой моды $v^{a}$. Эта мода не может быть “устранена” преобразованиями указанной калибровочной группы, так как непосредственно связана с инвариантом относительно этой группы $[8],[16]$

$$
\operatorname{Sp} P \exp \left(i g \int_{-L}^{L} d x^{-} \hat{A}_{-}\right)
$$

После наложения условия (9) остается следующий калибровочный произвол в выбоpe $v^{a}$ :

$$
\hat{v} \rightarrow \widetilde{\Omega}\left(\hat{v}+\frac{\pi l}{g L} \hat{n}\right) \widetilde{\Omega}^{-1}, \quad n^{a} \equiv \frac{v^{a}}{\sqrt{v^{a^{\prime}} v^{a^{\prime}}}}, \quad l=0, \pm 1, \pm 2, \ldots
$$

Он соответствует преобразованиям

$$
\Omega(x)=\widetilde{\Omega}\left(x^{\perp}, x^{+}\right) e^{i \hat{n} \pi l x^{-} / L} .
$$

Матрицы (12) тесно связаны с топологической классификацией классических калибровочных полей в замкнутом по $x^{-}$пространстве-времени [8]; нетривиальным классам отвечают такие функции $v^{a}\left(x^{\perp}, x^{+}\right)$, которые нельзя с помощью калибровочного преобразования матрицами вида (12) топологически непрерьвно направить вдоль калибровочной оси “3” в окрестности особых точек, где $v^{a}=n^{a} \pi l /(g L)$. В дальнейшем мы оставляем в стороне интересный вопрос о роли таких классов потенциалов [8] и будем рассматривать только тривиальньй класс [9], [16], которьй допускает калибровку

$$
v^{a}=\delta^{a 3} v, \quad A_{-}^{1}=A_{-}^{2}=0, \quad A_{-}^{3} \equiv v .
$$

Это условие ограничивает калибровочный произвол до абелевой группы

$$
\Omega(x)=e^{i \omega\left(x^{\perp}, x^{+}\right) \tau^{3} / 2}
$$

и группы преобразований вида $(12)$ с $n^{a}=\delta_{a 3}, \widetilde{\Omega} \equiv 1$.

Введем комбинации потенциалов, просто преобразуюшиеся относительно (14):

$$
\begin{array}{ll}
W_{\mu} \equiv \frac{A_{\mu}^{1}+i A_{\mu}^{2}}{\sqrt{2}}, & W_{\mu}^{+} \equiv \frac{A_{\mu}^{1}-i A_{\mu}^{2}}{\sqrt{2}}, \\
W_{\mu} \stackrel{\omega}{\longrightarrow} W_{\mu} e^{-i \omega}, & A_{\mu}^{3} \stackrel{\omega}{\longrightarrow} A_{\mu}^{3}+\frac{1}{g} \partial_{\mu} \omega .
\end{array}
$$


В силу условий периодичности их можно представить в виде рядов Фурье:

$$
\begin{aligned}
A_{\mu}^{3}(x) & =\frac{1}{\sqrt{2 L}} \sum_{n} A_{n \mu}\left(x^{\perp}, x^{+}\right) e^{-i p_{n} x^{-}}, \\
W_{\mu}(x) & =\frac{1}{\sqrt{2 L}} \sum_{n} W_{n \mu}\left(x^{\perp}, x^{+}\right) e^{-i p_{n} x^{-}}, \\
\Psi^{i}(x) & =\frac{1}{\sqrt{2 L}} \sum_{n} \Psi_{n}^{i}\left(x^{\perp}, x^{+}\right) e^{-i p_{n} x^{-}},
\end{aligned}
$$

где $p_{n}=\pi n / L$, для периодических условий $p_{n}^{\prime}=p_{n}$, а для антипериодических $p_{n}^{\prime}=$ $p_{n+1 / 2}$.

Обратимся теперь к лагранжиану (8) и выразим его через переменные (16) с учетом калибровки (13). Получаем следуюшее выражение [16]:

$$
\begin{aligned}
\int_{-L}^{L} \mathcal{L} d x^{-}= & L\left(\partial_{+} v\right)^{2}-\sqrt{2 L} \partial_{+} A_{0 k} \partial_{k} v+\sum_{n>0} 2 i p_{n} A_{n k}^{+} \partial_{+} A_{n k}+ \\
& +\sum_{n=-\infty}^{\infty}\left[2 i\left(p_{n}-g v\right) W_{n k}^{+} \partial_{+} W_{n k}+i \sqrt{2} \chi_{n}^{i+} \partial_{+} \chi_{n}^{i}\right]+\mathcal{L}^{\prime}
\end{aligned}
$$

где $\mathcal{L}^{\prime}$ не содержит производных по “времени” $x^{+}$:

$$
\begin{aligned}
\mathcal{L}^{\prime} \equiv & \int_{-L}^{L} d x^{-}\left[\frac{1}{2}\left(D_{-} A_{+}\right)^{a}\left(D_{-} A_{+}\right)^{a}+A_{+}^{a}\left(D_{k} F_{-k}^{a}+\frac{g}{\sqrt{2}} \chi^{+} \tau_{a} \chi\right)-\right. \\
& \left.-\frac{1}{2} F_{12}^{a} F_{12}^{a}+i \sqrt{2} \xi^{+} \widehat{D}_{-} \xi+\left\{i \xi^{+}(\bar{D}-M) \chi+\text { э.с. }\right\}\right] .
\end{aligned}
$$

Введем канонически сопряженные "импульсы" к динамическим переменным $v, A_{n k}$ $(n>0), W_{n k}, \chi_{n}^{i}(-\infty<n<\infty)$ :

$$
\begin{aligned}
\Pi & \equiv \frac{\partial \mathcal{L}}{\partial\left(\partial_{+} v\right)}=2 L \partial_{+} v-\sqrt{2 L} \partial_{k} A_{0 k}, \\
\Pi_{n k}^{3} & \equiv \frac{\partial \mathcal{L}}{\partial\left(\partial_{+} A_{n k}\right)}=2 i p_{n} A_{n k}^{+}, \quad n>0 \\
\Pi_{n k} & \equiv \frac{\partial \mathcal{L}}{\partial\left(\partial_{+} W_{n k}\right)}=2 i\left(p_{n}-g v\right) W_{n k}^{+}, \\
\pi_{n}^{i} & \equiv \frac{\partial \mathcal{L}}{\partial\left(\partial_{+} \chi_{n}^{i}\right)}=i \sqrt{2} \chi_{n}^{i+} .
\end{aligned}
$$

Остальные переменные $\xi, A_{n+}, W_{n+}, W_{n+}^{+}, A_{0 k}$ зависимы и определяются из связей, к которым приводит принцип наименњшего действия для лагранжиана (17).

Варьируя по $\xi^{+}$, получаем

$$
\sqrt{2} \widehat{D}_{-} \xi+(\bar{D}-M) \chi=0
$$


при варьировании по $A_{+}^{a}$ имеем

$$
\begin{gathered}
D_{-} D_{-} A_{+}^{a}=D_{k} F_{-k}^{a}+\frac{g}{\sqrt{2}} \chi^{+} \tau^{a} \chi \\
a=3: \partial_{-} \partial_{-} A_{+}^{3}=\partial_{-} \partial_{k} A_{k}^{3}-\sqrt{2 L} \partial_{k} \partial_{k} v+ \\
+i g\left[W_{k}\left(\partial_{-}-i g v\right) W_{k}^{+}-\text {э.c. }\right]+\frac{g}{\sqrt{2}} \chi^{+} \tau^{3} \chi
\end{gathered}
$$

или

$$
\begin{aligned}
a=1,2: \quad\left(\partial_{-}+i g v\right)^{2} W_{+}= & \left(\partial_{-}+i g v\right)\left[\left(\partial_{k}+i g A_{k}^{3}\right) W_{k}\right]- \\
& -2 i g\left(\partial_{-} A_{k}^{3}-\partial_{k} v\right) W_{k}+\frac{g}{\sqrt{2}} \chi^{+} \tau^{+} \chi \\
\tau^{+} \equiv & \frac{\tau^{1}+i \tau^{2}}{\sqrt{2}}
\end{aligned}
$$

варьирование по $A_{0 k}$ дает связь

$$
\begin{array}{r}
\left(\partial_{1}^{2}+\partial_{2}^{2}\right) A_{0 k}+\frac{\partial_{k} \Pi}{\sqrt{2 L}}+i g\left\{W_{k}^{+} F_{+-}-\varepsilon_{k k^{\prime}} W_{k^{\prime}} F_{12}-\right. \\
\left.-\varepsilon_{k k^{\prime}} \partial_{k^{\prime}}\left(W_{1}^{+} W_{2}\right)+\frac{i}{2} \xi^{+} \tau^{3} \sigma_{k} \chi-\text { э.с. }\right\}=0
\end{array}
$$

где $\varepsilon_{12}=-\varepsilon_{21}=1, F_{+-} \equiv-\left(\partial_{-}+i g v\right) W_{+}, F_{12} \equiv\left(\partial_{1}+i g A_{1}^{3}\right) W_{2}-\left(\partial_{2}+i g A_{2}^{3}\right) W_{1}$. Не определяемая из этих связей компонента $A_{0+}$ играет роль множителя Лагранжа при связи первого рода, генерируюшей калибровочные преобразования (14):

$$
-2 L \partial_{k} \partial_{k} v+i g\left(W_{k}\left(\partial_{-}-i g v\right) W_{k}^{+}-\text {э.c. }\right)_{0}+\frac{g}{\sqrt{2}}\left(\chi^{+} \tau_{3} \chi\right)_{0}=0
$$

Это выражение есть нулевая мода уравнения связи (21).

Выражение гамильтониана через канонические переменные имеет следуюший вид:

$$
\begin{aligned}
H= & \int d^{2} x\left\{\frac{1}{2}\left(\frac{\Pi}{\sqrt{2 L}}+\partial_{k} A_{0 k}\right)^{2}+\int_{-L}^{L} d x^{-}\left[\frac{1}{2}\left(F_{12}^{3}\right)^{2}+\frac{1}{2}\left(\partial_{-} A_{+}^{3}\right)^{2}+\right.\right. \\
& \left.\left.+\left(F_{12} F_{12}^{+}+F_{+-} F_{+-}^{+}+\text {э.с. }\right)+\frac{i}{\sqrt{2}} \chi^{+}(\bar{D}+M) \widehat{D}_{-}^{-1}(\bar{D}-M) \chi\right]\right\} .
\end{aligned}
$$

Здесь подразумевается, что переменные $A_{k}, W_{k}, F_{12}^{3}, A_{+}^{3}, F_{+-}, \chi$ выражены через канонические переменные $v, \Pi, A_{n k}, \Pi_{n k}^{3}(n>0), W_{n k}, \Pi_{n k} \quad(-\infty<n<\infty), \chi_{n}^{i}, \pi_{n}^{i}$ с помощью равенств (16), (19)-(23). Все перечисленные величины, кроме $A_{0 k}$, находятся из указанных равенств явно. Переменная $A_{0 k}$ должна определяться из сложного дифференциального уравнения (23), что весьма трудно.

Наряду с описанным формализмом можно рассмотреть модель, в которой периодические по $x^{-}$поля ограничиваются условием

$$
A_{-}^{a}=0, \quad a=1,2,3 .
$$


В этом случае имеет место глобальная $S U(2)$-симметрия, но число связей первого рода типа (24) и второго рода типа (23) возрастает (и они усложняются).

Отмеченные трудности обусловлены наличием нулевых мод. Они исчезают, если в исходном лагранжиане с самого начала отбросить все нулевые моды и далее развивать каноническую теорию на основе такого упрошенного лагранжиана. В этом случае канонический формализм вообше не содержит связей, но остаточная калибровочная инвариантность отсутствует.

\section{3. КВАНТОВАНИЕ КАЛИБРОВОЧНОЙ ТЕОРИИ В КООР ДИНАТАХ СВЕТОВОГО ФРОНТА}

Перейдем от классических канонических переменных к квантовым операторам с (анти)перестановочными соотношениями $\left(x^{+}=\right.$const $)$

$$
\begin{aligned}
{\left[v\left(x^{\perp}\right), \Pi\left(x^{\prime \perp}\right)\right] } & =\left[A_{n k}\left(x^{\perp}\right), \Pi_{n k}^{3}\left(x^{\prime \perp}\right)\right]=\left[W_{n k}\left(x^{\perp}\right), \Pi_{n k}\left(x^{\prime \perp}\right)\right]= \\
& =\left\{\chi_{n r}^{i}\left(x^{\perp}\right), \pi_{n r}^{i}\left(x^{\prime \perp}\right)\right\}=i \delta^{(2)}\left(x^{\perp}-x^{\prime \perp}\right)
\end{aligned}
$$

и остальными коммутаторами (либо антикоммутаторами $\{\cdot, \cdot\}$ для фермионов), равными нулю.

После перехода к квантовым величинам легко найти низшее собственное состояние оператора $P_{-}$. Всегда можно выбрать такой порядок операторов канонических переменных ( "нормальный" порядок), чтобы минимальное собственное значение $P_{-}$равнялось нулю. Низшее собственное состояние оператора $P$ - играет роль фоковского вакуума. Можно ввести соответствуюшие фоковские операторы рождения и уничтожения: $a_{n k}^{+}$и $a_{n k}(n>0), w_{n k}^{+}$и $w_{n k}(-\infty<n<\infty), \varkappa_{n r}^{i}{ }^{+}$и $\varkappa_{n r}^{i}(-\infty<n<\infty)$, которые связаны с каноническими переменными:

$$
\begin{gathered}
a_{n k}=\sqrt{2 p_{n}} A_{n k}, \quad a_{n k}^{+}=\frac{\Pi_{n k}^{3}}{i \sqrt{2 p_{n}}}, \quad n>0, \\
w_{n k}=\theta\left(p_{n}-g v\right) \sqrt{2\left|p_{n}-g v\right|} W_{n k}+\frac{\theta\left(-p_{n}+g v\right)}{i \sqrt{2\left|p_{n}-g v\right|} \Pi_{n k},} \\
w_{n k}^{+}=\frac{\theta\left(p_{n}-g v\right)}{i \sqrt{2\left|p_{n}-g v\right|}} \Pi_{n k}-\theta\left(-p_{n}+g v\right) \sqrt{2\left|p_{n}-g v\right|} W_{n k}, \\
\varkappa_{n r}=\theta\left(p_{n}+g \hat{v}\right) 2^{1 / 4} \chi_{n r}+\theta\left(-p_{n}-g \hat{v}\right) 2^{1 / 4} \frac{\pi_{n r}}{i \sqrt{2}}, \\
\varkappa_{n r}^{+}=\theta\left(p_{n}+g \hat{v}\right) 2^{1 / 4} \frac{\pi_{n r}}{i \sqrt{2}}+\theta\left(-p_{n}-g \hat{v}\right) 2^{1 / 4} \chi_{n r},
\end{gathered}
$$

где $\theta(x)=1$ при $x>0, \theta(x)=0$ при $x<0$, и удовлетворяют стандартным перестановочным соотношениям. В терминах этих операторов рождения и уничтожения импуль- 
сы $P_{-}$и $P_{k}$ имеют такой же вид, как и в теории невзаимодействуюших полей:

$$
\begin{aligned}
P_{-}= & \int d^{2} x\left(2 L \partial_{k} v \partial_{k} v+\sum_{n>0} a_{n k}^{+} a_{n k} p_{n}+\right. \\
& \left.+\sum_{n=-\infty}^{\infty}\left(w_{n k}^{+} w_{n k}\left|p_{n}-g v\right|+\varkappa_{n r}^{+}\left|p_{n}+g \hat{v}\right| \varkappa_{n r}\right)\right), \\
P_{k}= & \int d^{2} x\left(A_{0 k} Q+\Pi \partial_{k} v+\sum_{n>0} a_{n k^{\prime}}^{+} i \partial_{k} a_{n k^{\prime}}+\right. \\
& \left.+\sum_{n=-\infty}^{\infty}\left(w_{n k^{\prime}}^{+} i \partial_{k} w_{n k^{\prime}}+\varkappa_{n r}^{i+} i \partial_{k} \varkappa_{n r}^{i}\right)\right),
\end{aligned}
$$

где $Q$ - оператор связи, соответствуюший выражению, стоящему в левой части уравнения (24):

$$
Q \equiv-2 L \partial_{k} \partial_{k} v-\sum_{n=-\infty}^{\infty}\left(w_{n k}^{+} w_{n k} g \varepsilon\left(p_{n}-g v\right)+\varkappa_{n r}^{+} \frac{\tau^{3}}{2} g \varepsilon\left(p_{n}+g \hat{v}\right) \varkappa_{n r}\right),
$$

а $\varepsilon\left(p_{n}-g v\right)-$ знаковая функция.

Наложим связь (29) на допустимые физические векторы состояний [17]. Это условие означает их калибровочную инвариантность относительно группы (14). В фоковском представлении векторы состояний образуются с помощью введенных операторов рождения. При калибровочных преобразованиях (14) эти операторы изменяются следующим образом:

$$
\begin{aligned}
& w_{n k}^{+} \stackrel{\omega}{\longrightarrow} w_{n k}^{+} e^{i \omega \varepsilon\left(p_{n}-g v\right)}, \\
& \varkappa_{n r}^{+} \stackrel{\omega}{\longrightarrow} \varkappa_{n r}^{+} e^{-i \omega \varepsilon\left(p_{n}+g \hat{v}\right) \tau^{3} / 2} .
\end{aligned}
$$

Поэтому фоковские векторы состояний оказываются, вообще говоря, калибровочно-неинвариантными. Чтобы получить калибровочно-инвариантные векторы, достаточно переопределить операторы рождения, умножая их на фазовый операторный множитель, построенный с помощью переменной $\pi$, которая преобразуется как величина $\partial_{k} A_{0 k}$. C этой целью определим оператор фазы $\eta$ :

$$
\eta(x)=-\frac{g}{2 L}\left(\partial_{k} \partial_{k}\right)^{-1} \Pi(x)=\frac{g}{4 \pi L} \int d^{2} x^{\prime} \ln \left|x^{\perp}-x^{\prime}\right| \Pi\left(x^{\prime}\right),
$$

преобразуюшийся по закону $\eta \stackrel{\omega}{\longrightarrow} \eta-\omega$. Тогда калибровочно-инвариантные операторы рож дения принимают следующий вид:

$$
w_{n k}^{+} e^{i \varepsilon\left(p_{n}-g v\right) \eta}, \quad \varkappa_{n r}^{+} e^{-i \varepsilon\left(p_{n}+g \hat{v}\right) \eta \tau^{3} / 2} .
$$

Отметим, что в силу связи (29) фоковский вакуум есть собственное состояние оператора $v$ c собственным значением, удовлетворяюшим уравнению $\partial_{k} \partial_{k} v(x)=0$. Предполагая, что потенциалы $A_{\mu}^{a}$ не возрастают при $x^{\perp} \rightarrow \infty$, получаем, что указанное собственное 
значение представляет собой постоянную функцию. Значение этой функции обозначим через $v_{0}$. Поскольку величина П, определяющая фазу $\eta$, канонически сопряжена с переменной $v$, то фазовые множители в выражениях (31) действуют на собственные состояния $v$ как операторы сдвига. Они сдвигают собственное значение $v(x)$ на величину

$$
-\frac{g}{4 \pi L} \varepsilon\left(p_{n}-g v\left(x_{1}\right)\right) \ln \left|x^{\perp}-x_{1}^{\perp}\right|
$$

для оператора $w_{n k}^{+}\left(x_{1}\right)$ и на величину

$$
\frac{g}{4 \pi L}\left(\varepsilon\left(p_{n}+g \hat{v}\left(x_{1}\right)\right) \frac{\tau^{3}}{2}\right)_{i i} \ln \left|x^{\perp}-x_{1}^{\prime \perp}\right|
$$

для оператора $\varkappa_{n r}^{i+}\left(x_{1}^{\prime}\right)$. Отсюда следует, что калибровочно-инвариантный фоковский вектор получается применением операторов рождения $w_{n k}^{+}, \varkappa_{n r}^{i+}$ к собственному состоянию оператора $v$, собственное значение которого отличается от вакуумного $\left(v_{0}\right)$ на сумму сдвигов (32), (33), т.е. равно

$$
\begin{aligned}
v(x)= & v_{0}-\frac{g}{4 \pi L} \sum_{l} \varepsilon\left(p_{n_{l}}-g v\left(x_{l}\right)\right) \ln \left|x^{\perp}-x_{l}^{\perp}\right|+ \\
& +\frac{g}{4 \pi L} \sum_{l^{\prime}} \varepsilon\left(p_{n_{l^{\prime}}}+g \hat{v}_{i_{l^{\prime}} i_{l^{\prime}}}\left(x_{l^{\prime}}^{\prime}\right)\right) \frac{\tau_{i_{l^{\prime}} i_{l^{\prime}}}^{3} \ln \left|x^{\perp}-x_{l^{\prime}}^{\perp}\right| .}{2}
\end{aligned}
$$

Асимптотика этого собственного значения при $x^{\perp} \rightarrow \infty$ определяется условием равенства нулю полного “заряда" всех "частиц" (т.е. "глюонов", рожденных в точках $x_{l}^{\perp}$, и “кварков", рожденных в точках $x_{l^{\prime}}^{\prime \perp}$ ):

$$
q \equiv \sum_{l} g \varepsilon\left(p_{n_{l}}-g v\left(x_{l}\right)\right)-\sum_{l^{\prime}} g \varepsilon\left(p_{n_{l^{\prime}}}+g \hat{v}_{i_{l^{\prime}}{ }^{\prime}{ }_{l^{\prime}}}\left(x_{l^{\prime}}^{\prime}\right)\right) \frac{\tau_{i_{l^{\prime}} i_{l^{\prime}}}^{3}}{2}=0 .
$$

Обсудим также особенности $v(x)$ при $x^{\perp} \rightarrow x_{l}^{\perp}$ или $x^{\perp} \rightarrow x_{l^{\prime}}^{\perp}$. Согласно формуле (34) мы формально получаем противоречие для знака $g v\left(x_{l}\right)$. Действительно, при $x^{\perp} \rightarrow x_{l}^{\perp}$ или $x^{\perp} \rightarrow x_{l^{\prime}}^{\prime}$, где $l$ либо $l^{\prime}$ - одно из значений, по которым ведется суммирование в формуле (34), там преобладает только один член, в котором зависимость от $p_{n_{l}}$ или $p_{n_{l^{\prime}}}$ перестает играть роль. При этом левая и правая части формулы (34) всегда имеют противоположные знаки. Этого можно избежать, только сосредоточивая в каждой точке $x_{l}$ несколько "частиц" с противоположными "зарядами" (тогда $v(x)=v_{0}$ ). Однако локализация нескольких частиц в одной точке обычно ведет в теории поля $\mathrm{k}$ ультрафиолетовым расходимостям энергии [18]. Поэтому необходима регуляризация $\left|x^{\perp}-x_{l}^{\perp}\right| \geqslant \Lambda^{-1}$, которая дает возможность исключить расходимость за счет перенормировки константы взаимодействия $g(\Lambda)[18]$. В самом деле, при $x^{\perp} \rightarrow x_{l}^{\perp}$ в этом случае имеем

$$
g v\left(x_{l}\right) \simeq g v_{0}+\frac{g^{2}(\Lambda)}{4 \pi L} \varepsilon\left(p_{n}-g v\left(x_{l}\right)\right) \ln \Lambda+O\left(g^{2}(\Lambda)\right) .
$$


Здесь предполагается, что $g^{2}(\Lambda) \rightarrow 0$ при $\Lambda \rightarrow \infty$ (как получается в обычной теории возмушений [19]). Если еше предположить, что $g^{2}(\Lambda) \ln (\Lambda) \rightarrow$ const (тоже в соответствии с ренормгруппой в теории возмущений [19]), то получим

$$
g v\left(x_{l}\right) \simeq g v_{0}+\frac{\text { const }}{4 \pi L} \varepsilon\left(p_{n}-g v_{0}\right) .
$$

Аналогично для фермионов

$$
g v\left(x_{l}\right) \simeq g v_{0}+\frac{\text { const }}{8 \pi L} \varepsilon\left(p_{n}-\frac{1}{2} g v_{0}\right) .
$$

Что касается значений $g v(x)$ в остальных точках, то они (при $\Lambda \rightarrow \infty)$ имеют порядок $g v_{0}+O\left(g^{2}(\Lambda)\right)$.

Величина $v_{0}$, определенная ранее для фоковского вакуума, может рассматриваться в физическом вакууме, отвечающем минимуму гамильтониана (25), как величина, квантовое значение которой соответствует низшему собственному состоянию оператора

$$
H_{0} \equiv\left\langle v_{0}|H| v_{0}\right\rangle=-(V)^{-1} \frac{d^{2}}{d v_{0}^{2}}+V U\left(v_{0}\right),
$$

где $V \equiv 2 L \int d^{2} x$ - объем пространства, $U\left(v_{0}\right)$ - плотность “потенциальной энергии". Функция $U\left(v_{0}\right)$ периодична с периодом $\pi /(g L)$ в силу остаточной калибровочной инвариантности относительно подстановки (11) и стремится к бесконечности при $v_{0} \rightarrow$ $\pi l /(g L)$. Минимуму $H_{0}$ соответствует такая неопределенность значения $v_{0}$, при которой особые точки $v_{0}=\pi l /(g L)$ фактически исключаются. При этом минимальное значение $H_{0}$ практически совпадает с минимумом "потенциальной энергии", так как $V \rightarrow \infty$.

Видно, что ультрафиолетовая регуляризация играет сушественную роль при рассмотрении квантовой теории. Поэтому в следуюших разделах мы опишем, как ввести ультрафиолетовую регуляризацию, не нарушая исходной калибровочной инвариантности, причем сделаем это для произвольной калибровочной групшы $U(N)$.

\section{4. КАЛИБРОВОЧНО-ИНВАРИАНТНОЕ ДЕЙСТВИЕ НА ПОПЕРЕЧНОЙ РЕШЕТКЕ}

Введем сначала частичную калибровочно-инвариантную ультрафиолетовую регуляризацию с помошью пространственной решетки по поперечным координатам $x^{1}, x^{2}$. При этом выберем переменные так, чтобы действие оказалось по ним полиномиальным [13]. Кроме того, мы используем описанную во введении калибровочно-инвариантную регуляризацию "б" сингулярностей при $p_{-} \rightarrow 0$ и калибровочно-инвариантное ультрафиолетовое обрезание по модам ковариантнойпроизводной $D_{-}$. После этого регуляризация становится полной. Для простоты рассматривается $U(N)$-теория одних лишь 

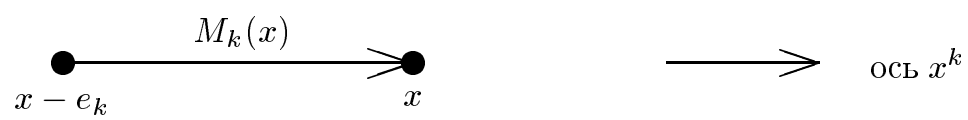

Рис. 1

векторных калибровочных полей, хотя обобщение на $S U(N)$-теорию или теорию с фермионами не представляет особого труда.

Компоненты калибровочного поля вдоль непрерывных координат $x^{+}, x^{-}$рассматриваются обычным образом, и мы их относим к узлам поперечной решетки. Компоненты поля вдоль поперечных координат описываются комплексными $(N \times N)$-матрицами $M_{k}(x), k=1,2$, которые отнесены к ребрам решетки. Эти матрицы не подчинены условиям унитарности, что и позволяет сделать действие полиномиальным. Матрица $M_{k}(x)$ относится к ребру, соединяющему узлы $x-e_{k}$ и $x$, и соответствует положительному направлению (вдоль оси $x^{k}$ ), вектор $e_{k}$ связывает соседние узлы решетки, он направлен вдоль оси $x^{k}$ и $\left|e_{k}\right|=a$ (см. рис. 1$)$. Матрица $M_{k}^{+}(x)$ относится к тому же самому ребру, но соответствует противоположному направлению (см. рис. 2).
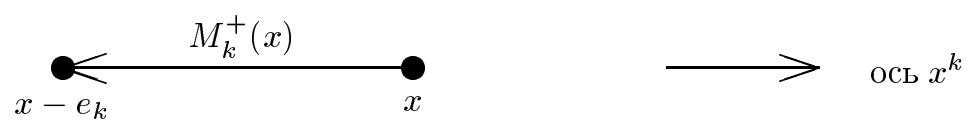

Рис. 2

Элементы этих матриц рассматриваются как независимые переменные. Любому замкнутому направленному циклу по ребрам решетки можно сопоставить след произведения матриц $M_{k}(x)$, расположенных на этих ребрах, упорядочив матрицы в согласии с последовательностью ребер в цикле справа налево. Например, выражение $\operatorname{Tr}\left\{M_{2}(x) \times\right.$ $\left.M_{1}\left(x-e_{2}\right) M_{2}^{+}\left(x-e_{1}\right) M_{1}^{+}(x)\right\}$ соответствует циклу, показанному на рис. 3 . Отметим, что цикл, состоящий из одного и того же ребра, пройденного в противоположных направлениях, не отвечает единице, так как матрицы не унитарны (см. рис. 4).

Калибровочные преобразования, осушествляемые унитарными матрицами $U(x)$, действуют на продольные компоненты поля как обычно (локально), а на матрицы $M$ и $M^{+}$ следуюшим образом:

$$
\begin{aligned}
M_{k}(x) \rightarrow M_{k}^{\prime}(x) & =U(x) M_{k}(x) U^{+}\left(x-e_{k}\right), \\
M_{k}^{+}(x) \rightarrow M_{k}^{\prime}(x) & =U\left(x-e_{k}\right) M_{k}^{+}(x) U^{+}(x) .
\end{aligned}
$$

Всякий след матрицы, отвечающей циклу (типа показанного на рис. 3,4 ), инвариантен относительно этих преобразований. Чтобы установить связь матричных переменных $M_{k}$ с обычными калибровочными полями, запишем эти переменные в виде

$$
M_{k}(x)=I+g a B_{k}(x)+i g a A_{k}(x), \quad B_{k}^{+}=B_{k}, \quad A_{k}^{+}=A_{k} .
$$




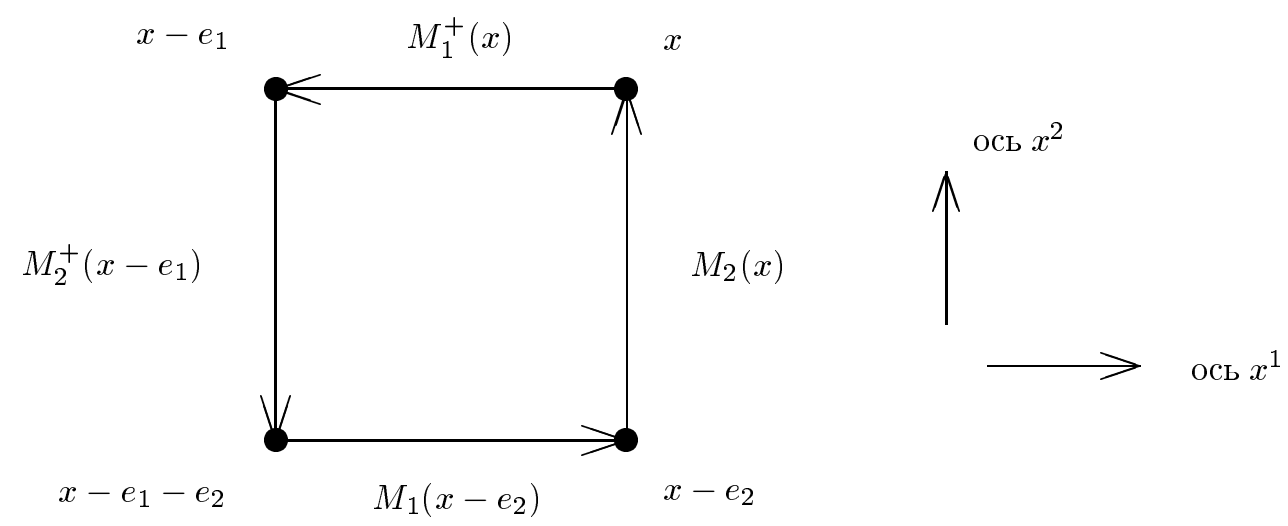

Рис. 3

Тогда в пределе $a \rightarrow 0$ поля $A_{k}(x)$ совпадают с поперечными компонентами калибровочного поля, а $B_{k}(x)$ оказываются добавочными (нефизическими) полями, которые должны отключаться в этом пределе. Ниже указано, как этого можно добиться.

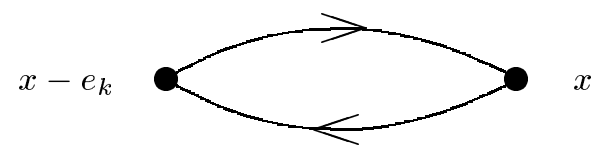

Рис. 4

Аналогом тензора напряженности калибровочного поля

$$
F_{\mu \nu}=\partial_{\mu} A_{\nu}-\partial_{\nu} A_{\mu}-i g\left[A_{\mu}, A_{\nu}\right]
$$

умноженного на $i$, являются величины

$$
\begin{aligned}
G_{+-} & =i F_{+-}, \quad F_{+-}(x)=\partial_{+} A_{-}(x)-\partial_{-} A_{+}(x)-i g\left[A_{+}(x), A_{-}(x)\right] \\
G_{ \pm k}(x) & =\frac{1}{g a}\left[\partial_{ \pm} M_{k}(x)-i g\left(A_{ \pm}(x) M_{k}(x)-M_{k}(x) A_{ \pm}\left(x-e_{k}\right)\right)\right] \\
G_{12}(x) & =-\frac{1}{g a^{2}}\left[M_{1}(x) M_{2}\left(x-e_{1}\right)-M_{2}(x) M_{1}\left(x-e_{2}\right)\right] .
\end{aligned}
$$

При калибровочных преобразованиях они изменяются следуюшим образом:

$$
\begin{aligned}
G_{+-}(x) & \rightarrow G_{+-}^{\prime}(x)=U(x) G_{+-}(x) U^{+}(x), \\
G_{ \pm k}(x) & \rightarrow G_{ \pm k}^{\prime}(x)=U(x) G_{ \pm k}(x) U^{+}\left(x-e_{k}\right), \\
G_{12}(x) & \rightarrow G_{12}^{\prime}(x)=U(x) G_{12}(x) U^{+}\left(x-e_{1}-e_{2}\right) .
\end{aligned}
$$


Рассматривается простейшая калибровочно-инвариантная форма действия, сводящаяся к обычной в пределе "наивного" перехода к непрерьвному пространству:

$$
\begin{aligned}
S= & a^{2} \sum_{x^{\perp}} \int d x^{+} \int_{-L}^{L} d x^{-} \operatorname{Tr}\left[G_{+-}^{+} G_{+-}+\right. \\
& \left.+\sum_{k}\left(G_{+k}^{+} G_{-k}+G_{-k}^{+} G_{+k}\right)-G_{12}^{+} G_{12}\right]+S_{m}
\end{aligned}
$$

где дополнительный член $S_{m}$ должен обеспечивать бесконечную массу полю $B_{k}$ в пределе $a \rightarrow 0$ :

$$
\begin{aligned}
S_{m}= & -\frac{m^{2}(a)}{4 g^{2}} \sum_{x^{\perp}} \int d x^{+} \int_{-L}^{L} d x^{-} \sum_{k} \operatorname{Tr}\left[\left(M_{k}^{+}(x) M_{k}(x)-I\right)^{2}\right] \underset{a \rightarrow 0}{\longrightarrow} \\
& \underset{a \rightarrow 0}{\longrightarrow}-m^{2}(a) \int d^{2} x^{\perp} \int d x^{+} \int_{-L}^{L} d x^{-} \sum_{k} \operatorname{Tr}\left(B_{k}^{2}\right), \quad m(a) \underset{a \rightarrow 0}{\longrightarrow} \infty
\end{aligned}
$$

Предполагается, что это приводит к отключению полей $B_{k}$.

\section{5. КАНОНИЧЕСКОЕ КВАНТОВАНИЕ НА СВЕТОВОМ ФРОНТЕ}

Выберем калибровочные условия в следуюшем виде:

$$
\partial_{-} A_{-}=0, \quad A_{-}^{i j}(x)=\delta^{i j} v^{j}\left(x^{\perp}, x^{+}\right) .
$$

Далее аргумент величин, не зависящих от $x^{-}$, для простоты также обозначается через $x$. Как уже было отмечено ранее, начав с произвольного поля $A_{\mu}^{i j}$, периодического по $x^{-}$, нельзя выбором калибровки сделать нулевую моду по $x^{-}$компоненты поля $A_{-}^{i j}$ равной нулю, не нарушая периодичности, но можно привести ее к диагональному виду [8]-[10]. Такой выбор калибровки и описывают условия (44).

Тогда действие (42) принимает вид

$$
\begin{aligned}
S= & a^{2} \sum_{x^{\perp}} \int d x^{+} \int_{-L}^{L} d x^{-}\left\{\sum_{i}\left[2 F_{+-}^{i i}(x) \partial_{+} v^{i}(x)\right]+\right. \\
& \left.+\frac{1}{(g a)^{2}} \sum_{i, j} \sum_{k}\left[D_{-} M_{k}^{i j+}(x) \partial_{+} M_{k}^{i j}(x)+\text { э.c. }\right]+\sum_{i, j} A_{+}^{i j}(x) Q^{j i}(x)-\mathcal{H}(x)\right\},
\end{aligned}
$$

где

$$
\begin{aligned}
D_{-} M_{k}^{i j}(x) & \equiv\left(\partial_{-}-i g v^{i}(x)+i g v^{j}\left(x-e_{k}\right)\right) M_{k}^{i j}(x), \\
D_{-} M_{k}^{i j+}(x) & \equiv\left(\partial_{-}+i g v^{i}(x)-i g v^{j}\left(x-e_{k}\right)\right) M_{k}^{i j+}(x), \\
D_{-} F_{+-}^{i j}(x) & \equiv\left(\partial_{-}-i g v^{i}(x)+i g v^{j}(x)\right) F_{+-}^{i j}(x),
\end{aligned}
$$


переменные $A_{+}^{i j}(x)$ играют роль множителей Лагранжа при связях

$$
\begin{aligned}
Q^{j i}(x) & \equiv 2 D_{-} F_{+-}^{j i}(x)+ \\
& +\frac{i}{g a^{2}} \sum_{j^{\prime}} \sum_{k}\left[M_{k}^{i j^{\prime}+}(x) D_{-} M_{k}^{j j^{\prime}}(x)-M_{k}^{j^{\prime} j+}\left(x+e_{k}\right) D_{-} M_{k}^{j^{\prime} i}\left(x+e_{k}\right)\right]=0
\end{aligned}
$$

$$
\mathcal{H}=\sum_{i j}\left(F_{+-}^{i j+} F_{+-}^{i j}+G_{12}^{i j+} G_{12}^{i j}\right)+\mathcal{H}_{m}
$$

есть плотность гамильтониана. Член $\mathcal{H}_{m}$ обычным образом получается из выражения (43).

Величины $F_{+-}^{i j}$ могут быть определены из связей (46), за исключением нулевых мод $F_{+-(0)}^{i i}$, которые не входят в эти связи и являются независимыми переменными. После этого остаются неразрешенными только нулевые моды связей $Q_{(0)}^{i i}\left(x^{\perp}, x^{+}\right)$, и мы накладываем соответствуюшие условия на физические состояния:

$$
Q_{(0)}^{i i}\left(x^{\perp}, x^{+}\right)\left|\Psi_{\text {phys }}\right\rangle=0 .
$$

Чтобы найти набор независимых канонических переменных, напишем фурье-преобразование полей $M_{k}^{i j}(x)$ по $x^{-}$в следуюшем виде:

$$
\begin{aligned}
M_{k}^{i j}(x)= & \frac{g}{\sqrt{4 L}} \sum_{n=-\infty}^{\infty}\left\{\Theta\left(p_{n}+g v^{i}(x)-g v^{j}\left(x-e_{k}\right)\right) M_{n k}^{i j}\left(x^{\perp}, x^{+}\right)+\right. \\
& \left.+\Theta\left(-p_{n}-g v^{i}(x)+g v^{j}\left(x-e_{k}\right)\right) M_{n k}^{i j+}\left(x^{\perp}, x^{+}\right)\right\} \times \\
& \times\left|p_{n}+g v^{i}(x)-g v^{j}\left(x-e_{k}\right)\right|^{-1 / 2} e^{-i p_{n} x^{-}}
\end{aligned}
$$

где

$$
\Theta(p)=\left\{\begin{array}{ll}
1, & p>0, \\
0, & p<0,
\end{array} \quad p_{n}=\frac{\pi}{L} n, \quad n \in \mathbb{Z} .\right.
$$

Из-за выбора калибровки (44) это фурье-преобразование совпадает с разложением по собственным модам оператора $D_{-}$, и, следовательно, применяемое нами далее ультраффиолетовое обрезание по модам этого оператора сводится к следуюшему условию, ограничивающему число членов в бесконечной сумме (49):

$$
\left|p_{n}+g v^{i}(x)-g v^{j}\left(x-e_{k}\right)\right| \leqslant \frac{\pi}{L} \bar{n},
$$

где $\bar{n}$ - целочисленный параметр ультраффиолетового обрезания. Подчеркнем, что такая регуляризация калибровочно-инвариантна.

С точностью до внеинтегральных членов действие переписывается следуюшим образом:

$$
\begin{aligned}
S= & a^{2} \sum_{x^{\perp}} \int d x^{+}\left\{\sum_{i} 4 L F_{+-(0)}^{i i} \partial_{+} v^{i}+\right. \\
& \left.+\frac{i}{a^{2}} \sum_{i, j} \sum_{k} \sum_{n}^{\prime} M_{n k}^{i j+} \partial_{+} M_{n k}^{i j}+2 L \sum_{i} A_{+(0)}^{i i} Q_{(0)}^{i i}-\widetilde{\mathcal{H}}(x)\right\},
\end{aligned}
$$


где $\sum_{n}^{\prime}{ }_{n}$ означает, что суммирование по $n$ ограничено условием (50), а плотность гамильтониана $\widetilde{\mathcal{H}}$ отличается от $\mathcal{H}$ подстановкой в (47) суммы

$$
F_{+-}^{i j}=\left(F_{+-}^{i j}-\delta^{i j} F_{+-(0)}^{i i}\right)+\delta^{i j} F_{+-(0)}^{i i},
$$

где выражение $F_{+-}^{i j}-\delta^{i j} F_{+-(0)}^{i i}$ должно быть записано в терминах переменных $M_{n k}^{i j}$, $M_{n k}^{i j+}, v^{i}$ путем использования связей (46) и разложения (49). Величины $G_{12}^{i j}$ также должны быть выражены через переменные $M_{n k}^{i j}, M_{n k}^{i j+}, v^{i}$. Выпишем набор пар канонически сопряженных переменных: $\left\{v^{i}, \Pi^{i}=4 L a^{2} F_{+-(0)}^{i i}\right\},\left\{M_{n k}^{i j}, i M_{n k}^{i j+}\right\}$. В квантовой теории эти переменные становятся операторами, удовлетворяюшими следуюшим перестановочным соотношениям:

$$
\begin{aligned}
{\left[v^{i}(x), \Pi^{j}\left(x^{\prime}\right)\right]_{x^{+}} } & =i \delta^{i j} \delta_{x^{\perp}, x^{\prime} \perp}, \\
{\left[M_{n k}^{i j}(x), M_{n^{\prime} k^{\prime}}^{i^{\prime} j^{\prime}+}\left(x^{\prime}\right)\right]_{x^{+}} } & =\delta^{i i^{\prime}} \delta^{j j^{\prime}} \delta_{n n^{\prime}} \delta_{k k^{\prime}} \delta_{x^{\perp}, x^{\prime} \perp} ;
\end{aligned}
$$

остальные коммутаторы равны нулю.

Заметим, что калибровочные условия (44) не фиксируют калибровку полностью. В частности, остается дискретная группа калибровочных преобразований, зависяших от $x^{-}$, вида

$$
U_{n}^{i j}(x)=\delta^{i j} \exp \left\{i \frac{\pi}{L} n^{i}\left(x^{\perp}\right) x^{-}\right\},
$$

где $n^{i}\left(x^{\perp}\right)$ - целые числа (наряду с группой непрерывных калибровочных преобразований, не зависяших от $x^{-}$). При преобразованиях (52) канонические переменные изменяются следуюшим образом:

$$
\begin{aligned}
v^{i}(x) & \rightarrow v^{i}(x)-\frac{\pi}{g L} n^{i}\left(x^{\perp}\right), \quad \Pi^{i} \rightarrow \Pi^{i}, \\
M_{n k}^{i j}\left(x^{\perp}\right) & \rightarrow M_{n^{\prime} k}^{i j}\left(x^{\perp}\right), \quad n^{\prime}=n+n^{i}\left(x^{\perp}\right)-n^{j}\left(x^{\perp}-e_{k}\right) .
\end{aligned}
$$

Выпишем выражение для квантового оператора $Q_{(0)}^{i i}\left(x^{\perp}, x^{+}\right)$, определяющего согласно (48) физическое подпространство состояний. Мы фиксируем порядок операторов так, чтобы классическим выражениям $G_{\mu \nu}^{+} G^{\mu \nu}$ всюду отвечали квантовые, упорядоченные по правилу $\left(G_{\mu \nu}^{+} G^{\mu \nu}+G^{\mu \nu} G_{\mu \nu}^{+}\right) / 2$. Отметим, что при другом способе упорядочения связи не допускают разумного вакуумного решения. В этом случае операторы $Q_{(0)}^{i i}\left(x^{\perp}, x^{+}\right)$в терминах канонических переменных имеют следуюший вид:

$$
\begin{aligned}
& 2 L Q_{(0)}^{i i}\left(x^{\perp}, x^{+}\right)=-\frac{g}{4 a^{2}} \sum_{j} \sum_{k} \sum_{n}^{\prime}\left[\varepsilon\left(p_{n}+g v^{j}\left(x+e_{k}\right)-g v^{i}(x)\right) \times\right. \\
& \quad \times\left(M_{n k}^{j i+}\left(x+e_{k}\right) M_{n k}^{j i}\left(x+e_{k}\right)+M_{n k}^{j i}\left(x+e_{k}\right) M_{n k}^{j i+}\left(x+e_{k}\right)\right)- \\
& \left.-\varepsilon\left(p_{n}+g v^{i}(x)-g v^{j}\left(x-e_{k}\right)\right)\left(M_{n k}^{i j+}(x) M_{n k}^{i j}(x)+M_{n k}^{i j}(x) M_{n k}^{i j+}(x)\right)\right],
\end{aligned}
$$

где

$$
\varepsilon(p)= \begin{cases}1, & p>0 \\ -1, & p<0\end{cases}
$$


Запишем выражение для светоподобного импульса $P_{-}$. Каноническое выражение генератора сдвига по $x^{-}$

$$
\begin{aligned}
P_{-}^{\text {can }}= & \frac{1}{2} \sum_{x^{\perp}} \sum_{i, j} \sum_{k} \sum_{n}^{\prime} p_{n} \varepsilon\left(p_{n}+g v^{i}(x)-g v^{j}\left(x-e_{k}\right)\right) \times \\
& \times\left(M_{n k}^{i j+}(x) M_{n k}^{i j}(x)+M_{n k}^{i j}(x) M_{n k}^{i j+}(x)\right)
\end{aligned}
$$

можно переписать в калибровочно-инвариантном виде, добавляя член, пропорциональньй связи:

$$
\begin{aligned}
P_{-}= & \frac{a^{2}}{2} \sum_{x^{\perp}} \sum_{k} \int_{-L}^{L} d x^{-} \operatorname{Tr}\left(G_{-k}^{+} G_{-k}+G_{-k} G_{-k}^{+}\right)= \\
= & P_{-}^{\mathrm{can}}+4 L a^{2} \sum_{x^{\perp}} \sum_{i} v^{i} Q_{(0)}^{i i}= \\
= & \frac{1}{2} \sum_{x^{\perp}} \sum_{i, j} \sum_{k} \sum_{n}^{\prime}\left|p_{n}+g v^{i}(x)-g v^{j}\left(x-e_{k}\right)\right| \times \\
& \times\left(M_{n k}^{i j+}(x) M_{n k}^{i j}(x)+M_{n k}^{i j}(x) M_{n k}^{i j+}(x)\right)= \\
= & \sum_{x^{\perp}} \sum_{i, j} \sum_{k} \sum_{n}^{\prime}\left|p_{n}+g v^{i}(x)-g v^{j}\left(x-e_{k}\right)\right|\left(M_{n k}^{i j+}(x) M_{n k}^{i j}(x)+\frac{1}{2}\right) .
\end{aligned}
$$

Выберем такое представление пространства состояний, в котором динамические переменные $v^{i}(x)$ являются операторами умножения. Тогда состояния будут нормируемыми функционалами (точнее, функциями, так как $x^{\perp}$ дискретно) от классических функций $v^{i}(x)$. Можно определить полное пространство состояний как прямое произведение фоковского пространства, в котором $M_{n k}^{i j+}(x)$ и $M_{n k}^{i j}(x)$ играют роль операторов рождения и уничтожения, и пространства функционалов $F[v]$. Будем называть $M$-вакуумом состояния вида $|0\rangle \cdot F[v]$, где $|0\rangle$ удовлетворяет уравнению

$$
M_{n k}^{i j}(x)|0\rangle=0,
$$

а $F$ - некоторый функционал. Произвольное состояние можно представить в форме линейной комбинации векторов $|\{m\} ; F\rangle$ вида

$$
\prod_{x^{\perp}} \prod_{i, j} \prod_{k} \prod_{n}^{\prime}\left(M_{n k}^{i j+}(x)\right)^{m_{n k}^{i j}(x)}|0\rangle \cdot F[v]
$$

с различными неотрицательными целочисленными функциями $m_{n k}^{i j}\left(x^{\perp}\right)$ и функционалами $F$. При желании можно задать полный ортонормированный набор таких функционалов. 
Из (56) видно, что состояние, отвечающее минимуму $P_{-}$, должно удовлетворять условиям (57), т.е. должно являться $M$-вакуумом. Среднее значение $P_{-}$по такому состоянию можно записать в виде

$$
\begin{aligned}
\left\langle 0 ; F\left|P_{-}\right| 0 ; F\right\rangle= & \frac{1}{2} \int \prod_{x^{\perp}} \prod_{i} d v^{i}(x) \times \\
& \times \sum_{x^{\perp}} \sum_{i, j} \sum_{k} \sum_{n}^{\prime}\left|p_{n}+g v^{i}(x)-g v^{j}\left(x-e_{k}\right)\right||F[v]|^{2} .
\end{aligned}
$$

Напомним, что здесь $\sum_{n}^{\prime}$ означает ограничение суммирования по $n$ условием $(50)$. Используя это ограничение и сдвигая индекс суммирования $n$ на целую часть величины $g L\left(v^{i}(x)-v^{j}\left(x-e_{k}\right)\right) / \pi$, можно показать, что в суммах по $n$ зависимость от $v^{i}$ сокращается и выражение (59) не зависит от $F[v]$, если этот функционал нормирован на единицу. Таким образом, светоподобный импульс $P_{-}$имеет минимум на всех $M$-вакуумах. Вычитая из $P$ - соответствующую константу, можно добиться того, чтобы на таких состояниях оператор светоподобного импульса обращался в нуль.

Легко видеть, что все $M$-вакуумы оказываются физическими состояниями, т.е. удовлетворяют условиям связи (48). В самом деле, из (54) находим, что на таких состояниях условия связи имеют вид

$$
\sum_{j} \sum_{k} \sum_{n}^{\prime}\left[\varepsilon\left(p_{n}+g v^{j}\left(x+e_{k}\right)-g v^{i}(x)\right)-\varepsilon\left(p_{n}+g v^{i}(x)-g v^{j}\left(x-e_{k}\right)\right)\right] F[v]=0
$$

и выполняются для любого $F[v]$, так как для каждого ребра в сумме (60) число положительных и отрицательных значений $\varepsilon$-функций одинаково. Для произвольных базисных векторов (58) аналогичные условия связи имеют вид

$$
\begin{aligned}
& \sum_{j} \sum_{k} \sum_{n}^{\prime} {\left[\varepsilon\left(p_{n}+g v^{j}\left(x+e_{k}\right)-g v^{i}(x)\right) m_{n k}^{i j}\left(x+e_{k}\right)-\right.} \\
&\left.-\varepsilon\left(p_{n}+g v^{i}(x)-g v^{j}\left(x-e_{k}\right)\right) m_{n k}^{i j}(x)\right] F[v]=0 .
\end{aligned}
$$

Собственные значения $p_{-}$оператора $P_{-}$должны находиться из уравнения

$$
\sum_{x^{\perp}} \sum_{i, j} \sum_{k} \sum_{n}^{\prime}\left|p_{n}+g v^{i}(x)-g v^{j}\left(x-e_{k}\right)\right| m_{n k}^{i j}(x) F[v]=p_{-} F[v]
$$

с учетом требования нормируемости функционала $F[v]$.

Чтобы определить физический вакуум, надо рассматривать не только состояния, отвечаюшие минимуму оператора $P_{-}$, но и находить минимум гамильтониана $P_{+}$. Можно попытаться это сделать, минимизируя гамильтониан на $M$-вакуумах, отвечающих минимуму оператора $P_{-}$. Выражение $\left\langle 0\left|P_{+}\right| 0\right\rangle$, где $|0\rangle-$ фоковский вакуум по отношению к операторам $M_{n k}, M_{n k}^{+}$, зависит от функций $v^{i}(x)$, которые входят, в частности, в нормальные свертки операторов $M_{k}(x), M_{k}^{+}(x)$, и от операторов $\Pi^{i}(x)$, канонически 
сопряженных к переменным $v^{i}(x)$. Нужно минимизировать среднее значение этого выражения на пространстве функционалов $F[v]$. Результируюший функционал $F[v]$ должен быстро убывать вблизи тех значений $v^{i}(x)$, при которых оператор $D_{-}$имеет нулевое собственное значение, так как при этом гамильтониан сингулярен (это, например, видно из разложения (49)).

Построенный таким образом вакуум сильно отличается от вакуума теории в непрерывном пространстве, поскольку в построенном вакууме равны нулю не средние значения операторов $\left(M_{k}-I\right) /(g a)=B_{k}+i A_{k}$, связанных с обычным калибровочным полем, а средние значения операторов $M_{k}$. При этом трудно выполнить и условие унитарности матриц $M_{k}$ в пределе непрерьвного пространства (или эквивалентное условие отключения нефизических полей $B_{k}$ ). Причина такого несоответствия в отсутствии явной лоренц-инвариантности данной формулировки теории, вследствие чего квантовые состояния, отвечающие минимумам операторов $P_{+}$и $P_{-}$, могут не совпадать. Вопрос состоит в том, можно ли так определить переход к непрерывному пространству, чтобы указанные состояния стремились друг к другу хотя бы в этом пределе. Данная проблема требует дополнительного исследования.

Рассмотренное выше квантование на СФ может помочь усовершенствовать эффективные низкоэнергетические модели, основанные на аналогичном формализме (но в существующих вариантах не учитывающие нулевых мод и не использующие калибровочно-инвариантной регуляризации ультрафиолетовых расходимостей по $p_{-}$). Такие модели описаны, например, в статьях [15], где использована идея работ [20].

\section{6. ЗАКЛЮЧЕНИЕ}

В настояшей работе с помошњю решеточного формализма осушествлена калибровочно-инвариантная регуляризация теории поля на СФ в рамках гамильтонова подхода. Это сделано без использования теории возмушений, что не удавалось ранее. Однако возникли существенные трудности, обусловленные нарушением явной лоренц-инвариантности и наличием нефизических полей, требующих отключения в пределе снятия регуляризации. В настояшее время неясно, в какой мере удастся преодолеть эти трудности в случае обычной калибровочной теории. Независимо от этого данный подход может применяться для формулировки полуфеноменологических низкоэнергетических калибровочно-инвариантных моделей.

Благодарности. Работа С. А. Пастона поддержана Администрацией Санкт-Петербурга и Министерством образования России (грант № PD03-1.2-35). Авторы выражают благодарность региональному бюро ЮНЕСКО по научному сотрудничеству в Европе за поддержку Международной школы физики им. В. А. Фока.

\section{Список литературы}

[1] P. A. M. Dirac. Rev. Mod. Phys. 1949. V. 21. P. 392.

[2] S.-J. Chang, R. G. Root, T.-M. Yan. Phys. Rev. D. 1973. V. 7. P. 1133; F. Lenz, M. Thies, S. Levit, K. Yazaki. Ann. Phys. 1991. V. 208. P. 1. 
[3] Е. В. Прохватилов, В. А. Франке. ЯФ. 1989. Т. 49. № 4. С. 1109; 1996. Т. 59. № 11. C. 2105; E. V. Prokhvatilov, H. W. L. Naus, H.-J. Pirner. Phys. Rev. D. 1995. V. 51. P. 2933; hep-ph/9406275.

[4] M. Burkardt, A. Langnau. Phys. Rev. D. 1991. V. 44. P. 1187; P. 3857; M. Burkardt. Adv. Nucl. Phys. 1996. V. 23. P. 1.

[5] С. А. Пастон, В. А. Франке. ТМФ. 1997. Т. 112. № 3. С. 399; hep-th/9901110; S. A. Paston, E. V. Prokhvatilov, V. A. Franke. Nucl. Phys. B. Proc. Suppl. 2002. V. 108. № 1-3. P. 189; hep-th/0111009.

[6] С. А. Пастон, Е. В. Прохватилов, В. А. Франке. ТМФ. 1999. Т. 120. № 3. С. 417; hep-th/0002062; 2002. T. 131. № 1. C. 84; hep-th/0302016; S. A. Paston, E. V. Prokhvatilov, $V$. A. Franke. $\mathrm{QED}_{2}$ light-front Hamiltonian reproducing all orders of covariant chiral perturbative theory. hep-th/9910114; On the construction of corrected light-front Hamiltonian for $\mathrm{QED}_{2}$. hep-th/0011224.

[7] S. J. Brodsky, G. McCartor, H. C. Pauli, S. S. Pinsky. Part. World. 1993. V. 3. P. 109.

[8] V. A. Franke, Yu. V. Novozhilov, E. V. Prokhvatilov. Lett. Math. Phys. 1981. V. 5. P. 239.

[9] V. A. Franke, Yu. V. Novozhilov, E. V. Prokhvatilov. Lett. Math. Phys. 1981. V. 5. P. 437.

[10] V. A. Franke, Yu. V. Novozhilov, E. V. Prokhvatilov. Light-cone quantization of gauge theories with periodic boundary conditions. In: Dynamical Systems and Microphysics (Geometry and Mechanics). Eds. A. Avez, A. Blaquiere, A. Mazzollo. New York: Academic, 1982. P. 389.

[11] K. G. Wilson. Phys. Rev. D. 1974. V. 10. P. 2445.

[12] M. B. Halpern. Progr. Theor. Phys. Suppl. 1993. V. 111. P. 163.

[13] W. A. Bardeen, R. B. Pearson. Phys. Rev. D. 1976. V. 14. P. 547; W. A. Bardeen, R. B. Pearson, E. Rabinovici. Phys. Rev. D. 1980. V. 21. P. 1037; V. A. Franke, S. A. Paston, E. V. Prokhvatilov. Lattice gauge theories with polynomial action and their canonical formulation on the light front. hep-th/9803035.

[14] A. A. Andrianov, L. Bonora. Nucl. Phys. B. 1984. V. 233. P. 232; P. 247; A. A. Andrianov, Yu. V. Novozhilov. Phys. Lett. B. 1985. V. 153. P. 422.

[15] S. Dalley, B. van de Sande. Phys. Rev. D. 1997. V. 56. P. 7917; hep-ph/9704408; 1999. V. 59. P. 065008; hep-th/9806231; 2000. V. 62. P. 014507; hep-lat/9911035; 2003. V. 67. P. 114507; hep-ph/0212086.

[16] Ю. В. Новожилов, Е. В. Прохватилов, В. А. Франке. Вестн. ЛГУ. 1981. № 22. С. 13.

[17] П. А. М. Дирак. Принципы квантовой механики. М.: Наука, 1979.

[18] Н. Н. Боголюбов, Д. В. Ширков. Введение в теорию квантованных полей. М.: Наука, 1984.

[19] А. А. Славнов, Л. Д. Фаддеев. Введение в квантовую теорию калибровочных полей. М.: Наука, 1988.

[20] H.-J. Pirner. Progr. Part. Nucl. Phys. 1992. V. 29. P. 33; G. Mack. Nucl. Phys. B. 1984. V. 235. P. 197. 